\title{
MicroRNA-212 suppresses cell proliferation in nasopharyngeal carcinoma by targeting ELF3
}

\author{
YAOJIE KANG $^{1 *}$, YANFEI CUI $^{2 *}$ and MING TAN ${ }^{2}$ \\ Departments of ${ }^{1}$ Otolaryngology-Head and Neck Surgery, and ${ }^{2}$ Oncology, \\ Central Hospital of Enshi Autonomous Prefecture, Enshi, Hubei 445000, P.R. China
}

Received July 17, 2018; Accepted January 10, 2020

DOI: $10.3892 / \mathrm{ol} .2020 .11401$

\begin{abstract}
Nasopharyngeal carcinoma (NPC) is an epithelial malignancy of the head and neck that is prevalent in China. The present study investigated the molecular mechanisms of microRNA-212 (miR-212) and E74-like factor 3 (ELF3) in NPC cell lines and tissues. Using reverse transcription-quantitative PCR, the present study identified that miR-212 expression was downregulated in NPC cell lines and tissues. Furthermore, an elevated expression level of miR-212 was revealed to inhibit NPC cell proliferation, as determined using a cell counting kit-8 assay in vitro. ELF3 was identified as a direct target of miR-212 in NPC cells by a luciferase reporter assay. Additionally, the expression levels of miR-212 and ELF3 were negatively correlated in NPC tissues. The expression levels of ELF3 and miR-212 were associated with metastasis and TNM stage in patients with NPC. In summary, the present study indicated that miR-212 was downregulated in NPC and suppressed cell proliferation. This suggested that the miR-212/ELF3 axis may serve as a novel target for the diagnosis and treatment of NPC.
\end{abstract}

\section{Introduction}

Nasopharyngeal carcinoma (NPC) is an epithelial malignancy of the head and neck that is prevalent in China, with a high incidence rate (1). A number of factors are associated with the development and progression of NPC, including Epstein-Barr virus infection, genetic components and environmental factors (2). The prognosis of NPC is poor due to recurrence and distant metastasis; however, the use of chemoradiotherapy and intensity-modulated radiotherapy has increased the 5-year

Correspondence to: Dr Ming Tan, Department of Oncology, Central Hospital of Enshi Autonomous Prefecture, 158 Wuyang Road, Enshi, Hubei 445000, P.R. China

E-mail: tan0612ming@163.com

*Contributed equally

Key words: proliferation, microRNA-212, E74-like factor 3, nasopharyngeal carcinoma survival rate to $\sim 70 \%$ between January 2003 and December 2006 in Guangzhou, China $(3,4)$. Therefore, it is critical to understand the molecular mechanisms that underlie the progression of NPC, as this may promote the development of novel therapeutic strategies.

MicroRNAs (miRNAs/miRs) are a class of non-coding RNAs that are 19-25 nucleotides long $(5,6)$. Previous studies have demonstrated that miRNAs regulate a number of biological roles, including proliferation, apoptosis, migration and differentiation (7-9). Recent studies have demonstrated that miRNAs are abnormally expressed in numerous human diseases, particularly cancer. miRNAs can serve either as oncogenes or tumor suppressors during the progression of tumors $(7,9)$. A number of mature miRNAs have been demonstrated to be abnormally expressed in NPC, including miR-135a (10), miR-23a (11) and miR-203a-3p (12). This suggests that abnormally expressed miRNAs can contribute to the development and progression of NPC. miR-212 is abnormally expressed in numerous types of tumor, including non-small cell lung cancer (13), gastric cancer (14), NPC (15) and pancreatic cancer (16). Previous studies have indicated that miR-212 may serve a key role in cancer progression (13-16).

E74-like factor 3 (ELF3) is a transcription factor of the epithelial-specific subfamily, which serves an important role in a variety of pathophysiological processes in cancer (17). Previous studies have indicated that ELF3 is involved in cell proliferation, differentiation and migration in numerous types of human cancer. In colorectal cancer, ELF3 is overexpressed and promotes proliferation and invasion by enhancing $\beta$-catenin signaling (18). In hepatocellular carcinoma, ELF3 has been demonstrated to repress E-cadherin and promote the epithelial-mesenchymal transition by suppressing miR-141-3p, thereby activating zinc finger E-box binding homeobox 1 (19). However, to the best of our knowledge, the role of ELF3 in NPC remains unknown.

The present study demonstrated that miR-212 was downregulated in NPC cells and tissues. In addition, reduced expression levels of miR-212 were identified to be associated with clinical features of patients with NPC. Furthermore, overexpression of miR-212 suppressed cell proliferation in vitro. ELF3 was revealed to be downregulated by a direct interaction of its 3'-untranslated region (3'-UTR) with miR-212. The identified miR-212/ELF3 axis may provide novel evidence 
regarding the molecular mechanisms of NPC, and may reveal a novel therapeutic target for NPC.

\section{Materials and methods}

Tissues and cell lines. A total of 30 normal nasopharyngeal epithelial tissue specimens and paired freshly frozen NPC biopsy specimens were collected at the Rui Jin Hospital Affiliated to Shanghai Jiaotong University (Shanghai, China) between August 2016 and October 2017. The specimens were confirmed by histopathological examination. No patients with NPC had received radiotherapy or chemotherapy prior to biopsy. Written informed consent was obtained from all patients. The clinicopathological features and demographics of all patients are presented in Table I. The present study was approved by the Ethics Review Committee of Rui Jin Hospital Affiliated to Shanghai Jiaotong University (Shanghai, China).

The human immortalized nasopharyngeal epithelial cell line NP69 and the NPC cell line SUNE-1 were obtained from the Shanghai Cell Bank of Chinese Academy of Sciences. Each cell line was cultured in DMEM (Invitrogen; Thermo Fisher Scientific, Inc.) supplemented with 10\% FBS (HyClone; GE Healthcare Life Sciences) as well as $100 \mathrm{U} / \mathrm{ml}$ penicillin and $100 \mu \mathrm{g} / \mathrm{ml}$ streptomycin. All cells were maintained in a humidified incubator at $37^{\circ} \mathrm{C}$ with $5 \% \mathrm{CO}_{2}$.

Cell transfection. The miR-212 mimic, inhibitor, ELF3 small interfering RNA (siRNA) and negative controls (mimic-NC, inhibitor-NC or siRNA-NC) were obtained from Guangzhou RiboBio Co, Ltd. and transfected at a concentration of $100 \mathrm{nM}$. ELF3 plasmid and the empty control vector were purchased from Shanghai GenePharma Co., Ltd. NPC cells were grown in six-well plates and transfected with Lipofectamine ${ }^{\circledR} 2000$ (Invitrogen; Thermo Fisher Scientific, Inc.) according to the manufacturer's instructions. At $48 \mathrm{~h}$ post-transfection, cells were harvested for reverse transcription-quantitative PCR (RT-qPCR) analysis. The following sequences were used: miR-212 mimic (sense, 5'-ACCUUGGCUCUAGACUGC UUACU-3'; and antisense, 5'-UAAGCAGUCUAGAGCCAA GGUUU-3'), mimic negative control miRNA (sense, 5'-UUC UCCGAACGUGUCACGUTT-3'; and antisense, 5'-ACGUGA CACGUUCGGAGAATT-3'), miR-212 inhibitor (5'-GGCCGU GACUGGAGACUGUUA-3'), inhibitor-NC (5'-CAGUAC UUUUGUGUAGUACAA-3'), and ELF3 siRNA (5'-GCUACC AAGUGGAGAAGAATT-3') NC forward, 5'-UUCUCCGAA CGUGUCACGUTT-3' and reverse 5'-ACGUGACACGUU CGGAGAATT-3').

Additionally, ELF3 plasmid or empty vector was transfected into cells in the presence or absence of the miR-212 mimic using Lipofectamine 2000. The cells were then cultured for $24 \mathrm{~h}$ in six-well plates. The transfected cells were incubated in complete medium at $37^{\circ} \mathrm{C}$. RT-qPCR and western blot analysis were used to evaluate the transfection efficacy.

$R T$ - $q P C R$. Total RNA was extracted from the cultured cells and tissue samples using TRIzol ${ }^{\circledR}$ reagent (Invitrogen; Thermo Fisher Scientific, Inc.) according to the manufacturer's protocol. cDNA was synthesized using the SYBR PrimeScript RT-PCR kit (Takara Bio, Inc.) by incubating the mixture at $37^{\circ} \mathrm{C}$ for $15 \mathrm{~min}, 85^{\circ} \mathrm{C}$ for $5 \mathrm{sec}$ at $4^{\circ} \mathrm{C}$. When the temperature reaches $4^{\circ} \mathrm{C}$ this process ends. The ABI 7500 system (Applied Biosystems; Thermo Fisher Scientific, Inc.) was used for PCR and the thermocycling conditions were as follows: $95^{\circ} \mathrm{C}$ for $30 \mathrm{sec}$, followed by 40 cycles of $95^{\circ} \mathrm{C}$ for $5 \mathrm{sec}$ and $60^{\circ} \mathrm{C}$ for $34 \mathrm{sec}$. The relative expression was calculated via the comparative cycle threshold method and the levels of miR-212 and ELF3 were normalized using the U6 and GAPDH internal reference genes, respectively. The following primer sequences were used: miR-212 (forward, 5'-GGTAACAGTCTCCAG TCA-3' and reverse, 5'-GCAATTGCACTGGATACG-3'), U6 (forward, 5'-CTCGCTTCGGCAGCACA-3' and reverse, 5'-AACGCTTCACGAATTTGCGT-3'), GAPDH (forward, 5'-GCACCGTCAAGGCTGAGAAC-3' and reverse, 5'-TGG TGAAGACGCCAGTGGA-3'), ELF3 forward, (5'-GGCCCA GACCAAGCCTTAAT-3' and reverse, 5'-CACTGAAAGCCA GGGCAAAC-3'). Analysis of relative gene expression data was conducted using RT-qPCR and the $2^{-\Delta \Delta C q}$ method (20). All PCR experiments were performed in triplicate.

Western blot analysis. NPC cells were lysed and proteins were extracted with RIPA buffer (Beyotime Institute of Biotechnology). Subsequently, the concentrations of proteins were detected by using a BCA Protein Assay Kit (Beyotime Institute of Biotechnology). A total of $15 \mu \mathrm{g}$ of each protein sample were separated on $10 \%$ SDS-PAGE and transferred onto PVDF membranes. The membranes were blocked with $5 \%$ non-fat milk in TBST buffer for $2 \mathrm{~h}$ at room temperature, and incubated with primary antibodies against ELF3 (1:1,000; cat. no. AF6780; Beyotime Institute of Biotechnology) and GAPDH (1:2,000; cat. no. MB9231; Biogot Technology Co, Ltd.) overnight at $4^{\circ} \mathrm{C}$. Subsequently, the membranes were incubated with a secondary antibody conjugated to horseradish-peroxidase for $2 \mathrm{~h}$ at room temperature. The secondary antibody used for ELF3 was an anti-rabbit one (1:1,000; cat. no. A0208; Beyotime Institute of Biotechnology) and for GAPDH was an anti-mouse one $(1: 1,000$; cat. no. A0216; Beyotime Institute of Biotechnology). The targeted proteins in the membrane were detected using the Ncm-ECL Ultra kit (New Cell \& Molecular Biotech Co., Ltd). Western blotting data were analyzed using ImageJ (version 1.8.0; National Institutes of Health).

Luciferase assay. Site-directed mutagenesis was achieved in the miR-212 binding site of ELF3 mRNA using the QuikChange Lightning Site-Directed Mutagenesis kit (Agilent Technologies, Inc.) and TargetScan version 7.2 (http://www. targetscan.org/vert_72/) and miRanda (http://www.microrna. org/microrna/home.do) databases. The wild type (WT) or mutant (MUT) 3'-UTR fragment of ELF3 mRNA was then sub-cloned into a pGL3 luciferase vector (Promega Corporation) by PCR, which was co-transfected with either miR-212 mimic or mimic-NC into NPC cells for $36 \mathrm{~h}$ in 96-well plates using Lipofectamine 2000. Subsequently, a dual luciferase assay (Promega Corporation) was performed. Renilla (Promega Corporation) activity was used as the internal control.

Cell proliferation assay. Cell proliferation was determined using a cell counting kit-8 (CCK-8; Dojindo Molecular 
Table I. Association between clinicopathological characteristics and expression levels of miR-212 and ELF3 in nasopharyngeal carcinoma.

A, miR-212

\begin{tabular}{|c|c|c|c|c|c|}
\hline \multirow[b]{2}{*}{ Characteristics } & \multirow[b]{2}{*}{ Number, $\mathrm{n}$} & \multicolumn{2}{|c|}{ Expression level } & \multirow[b]{2}{*}{$\chi^{2}$} & \multirow[b]{2}{*}{ P-value } \\
\hline & & High, n (\%) & Low, n (\%) & & \\
\hline Sex & & & & 1.099 & 0.295 \\
\hline Male & 22 & $13(59.1)$ & $9(40.9)$ & & \\
\hline Female & 8 & $3(37.5)$ & $5(62.5)$ & & \\
\hline Age (years) & & & & 0.136 & 0.713 \\
\hline$\leq 45$ & 13 & $7(53.8)$ & $6(46.2)$ & & \\
\hline$>45$ & 17 & $8(47.1)$ & $9(52.9)$ & & \\
\hline Clinical stage & & & & 4.800 & 0.029 \\
\hline I-II & 10 & $6(60.0)$ & $4(40.0)$ & & \\
\hline III-IV & 20 & $4(20.0)$ & $16(80.0)$ & & \\
\hline Local or distant metastasis & & & & 8.623 & 0.003 \\
\hline No & 11 & $9(81.8)$ & $2(18.2)$ & & \\
\hline Yes & 19 & $5(26.3)$ & $14(73.7)$ & & \\
\hline
\end{tabular}

$\mathrm{B}, \mathrm{ELF} 3$

\begin{tabular}{|c|c|c|c|c|c|}
\hline \multirow[b]{2}{*}{ Characteristics } & \multirow[b]{2}{*}{ Number, $\mathrm{n}$} & \multicolumn{2}{|c|}{ Expression level } & \multirow[b]{2}{*}{$\chi^{2}$} & \multirow[b]{2}{*}{ P-value } \\
\hline & & High, n (\%) & Low, n (\%) & & \\
\hline Sex & & & & 2.058 & 0.151 \\
\hline Male & 22 & $12(54.5)$ & $10(45.5)$ & & \\
\hline Female & 8 & $2(25.0)$ & $6(75.0)$ & & \\
\hline Age (years) & & & & 1.033 & 0.310 \\
\hline$\leq 45$ & 13 & $6(46.2)$ & $7(53.8)$ & & \\
\hline$>45$ & 17 & $11(64.7)$ & $6(35.3)$ & & \\
\hline Clinical stage & & & & 6.429 & 0.011 \\
\hline I-II & 10 & $4(40.0)$ & $6(60.0)$ & & \\
\hline III-IV & 20 & $17(85.0)$ & $3(15.0)$ & & \\
\hline Local or distant metastasis & & & & 9.726 & 0.002 \\
\hline No & 11 & $3(27.3)$ & $8(72.7)$ & & \\
\hline Yes & 19 & $16(84.2)$ & $3(15.8)$ & & \\
\hline
\end{tabular}

ELF3, E74-like factor 3; miR-212, microRNA-212.

Technologies, Inc.) assay. Briefly, CCK-8 solution was added to the transfected cells in 96 -well plates $\left(1.0 \times 10^{3}\right.$ cells/well $)$ at days $0-5$ and incubated for $4 \mathrm{~h}$ at $37^{\circ} \mathrm{C}$. The absorbance of cells was detected at $450 \mathrm{~nm}$ using an ELISA plate reader (BioTek Instruments, Inc.). All experiments were performed in triplicate.

Statistical analysis. All data are presented as the mean \pm SEM. The experiments were repeated 3 times. Differences were assessed by a paired or unpaired Student's t-test for two group comparisons, as appropriate, one-way ANOVA followed by Tukey's post hoc test for multiple comparisons and Chi-square test. Pearson correlation analysis was used to determine the association between miR-212 and ELF3 expression. The statistical analyses were conducted using SPSS 18.0 software (SPSS, Inc.). $\mathrm{P}<0.05$ was considered to indicate a statistically significant difference.

\section{Results}

miR-212 is downregulated in NPC cells and tissues. First, the present study investigated the expression levels of miR-212 in tissue samples from patients with NPC to determine the role of miR-212 in NPC. As shown in Fig. 1A, the expression levels 
A

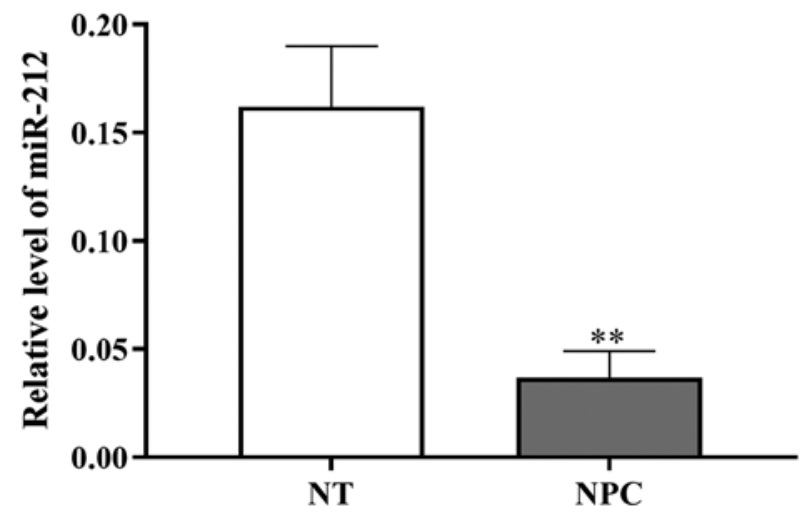

B

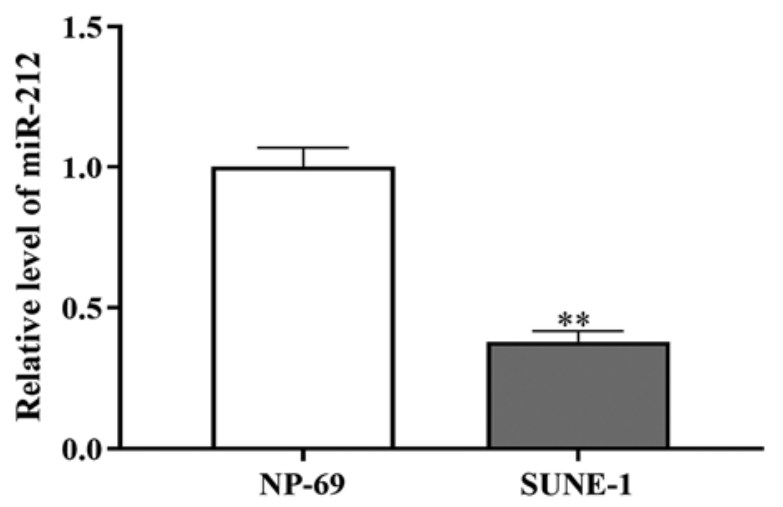

Figure 1. miR-212 expression is downregulated in NPC cell lines and tissues. (A) Relative expression levels of miR-212 in 30 NPC and adjacent normal tissues. U6 was used as an internal control. (B) miR-212 was significantly downregulated in the SUNE-1 cell line compared with the normal nasopharyngeal epithelial cell line NP69. ${ }^{* *} \mathrm{P}<0.01$ vs. the respective control group. miR-212, microRNA-212; NT, normal tissue; NPC, nasopharyngeal carcinoma.

A

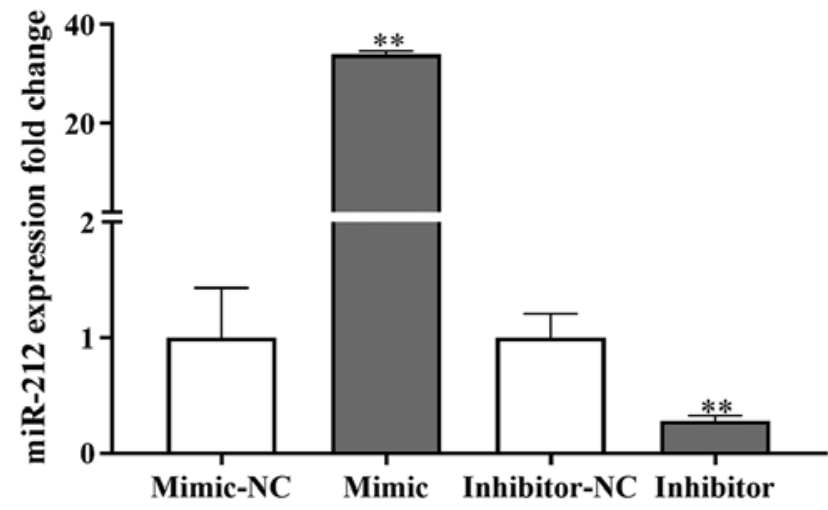

B

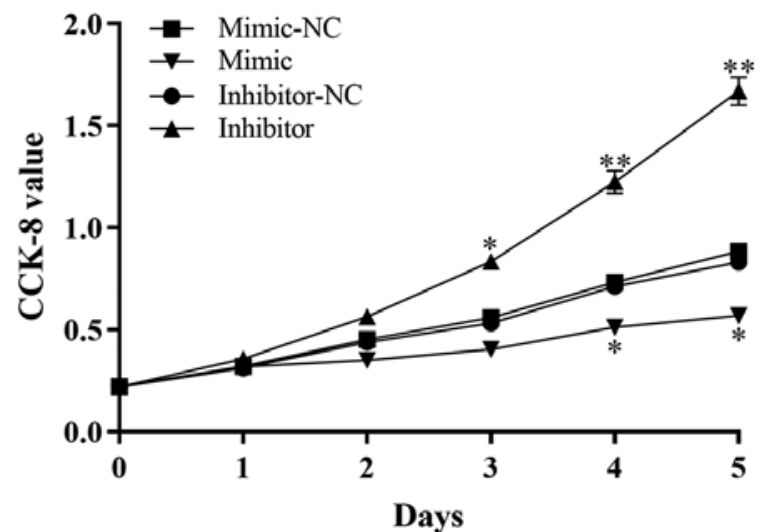

Figure 2. miR-212 inhibits NPC cell growth in vitro. (A) Overexpression and inhibition of miR-212 were confirmed by reverse transcription-quantitative PCR in SUNE-1 cells. (B) CCK-8 assays revealed that miR-212 mimic significantly inhibited cell growth, and miR-212 inhibitor significantly increased cell growth. ${ }^{*} \mathrm{P}<0.05,{ }^{* *} \mathrm{P}<0.01$ vs. the respective NC group. CCK-8, Cell Counting kit-8; miR-212, microRNA-212; NC, negative control.

of miR-212 were identified to be significantly lower in NPC tissue samples compared with normal tissue samples.

In addition, the expression level of miR-212 in the human NPC cell line SUNE-1 was investigated. RT-qPCR revealed that miR-212 expression was significantly downregulated in SUNE-1 cells compared with the normal nasopharyngeal epithelial cell line NP69 (Fig. 1B). In summary, miR-212 was revealed to be downregulated in NPC cells and tissues.

miR-212 inhibits NPC cell proliferation in vitro. miR-212 mimic, inhibitor and negative controls were used to evaluate the potential effects of miR-212 on NPC cell proliferation in vitro. The SUNE-1 cell line was selected as these cells demonstrated significantly different miR-212 expression levels compared with normal cells (Fig. 1B). Using RT-qPCR, the efficiency of miR-212 mimic and inhibitor transfection was assessed. Compared with negative controls, the miR-212 mimic significantly enhanced the expression levels of miR-212, and the miR-212 inhibitor significantly reduced the expression levels of miR-212 in SUNE-1 cells (Fig. 2A).
A CCK-8 assay was performed to examine the effect of miR-212 on the proliferation of SUNE-1 cells. The results revealed that miR-212 mimic significantly inhibited proliferation and the miR-212 inhibitor significantly increased proliferation compared with negative controls (Fig. 2B). These results indicated that miR-212 may suppress cell proliferation in NPC.

ELF3 is a direct target of miR-212 in NPC. To further elucidate the underlying mechanism of miR-212 in NPC cells, possible targets of miR-212 were predicted using the TargetScan and miRanda (http://www.microrna.org/microrna/home.do) databases. To further confirm the predictions, a luciferase reporter assay was performed in SUNE-1 cells. 3'-UTR regions of ELF3, which contained the predicted WT binding site of miR-212 or the MT site, were cloned into a luciferase vector (Fig. 3A). The results revealed that overexpression of miR-212 significantly decreased the luciferase activity of the WT ELF3 3'-UTR (Fig. 3B), whereas no effect was observed for the MT ELF3 3-UTR. In addition, transfection with miR-212 inhibitor increased the luciferase activity of WT ELF3 3'-UTR 
A

Site:333-340 of ELF3

3'UTR WT

hsa-miR-212-5P

5'-. . A AGACACCUGGACUGAGCCAAGGA. . .

3'UTR MT

3'-

UCAUUCGUCAGAUCUÇGUUUCCA

5'- AGACACCUGGACUGACCGAUGCU

B

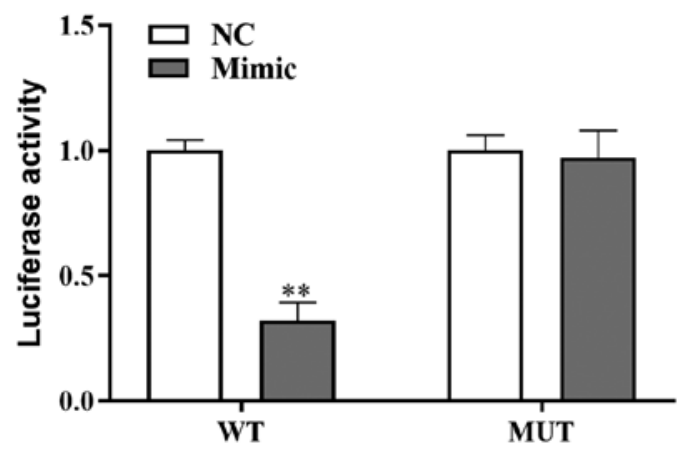

D

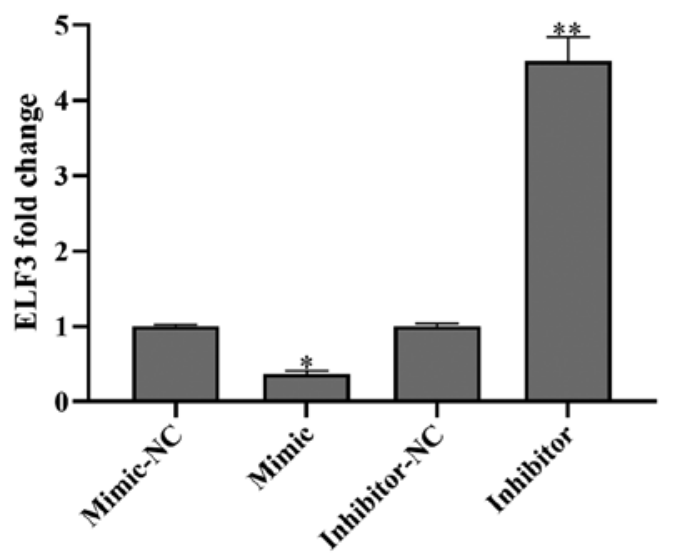

F

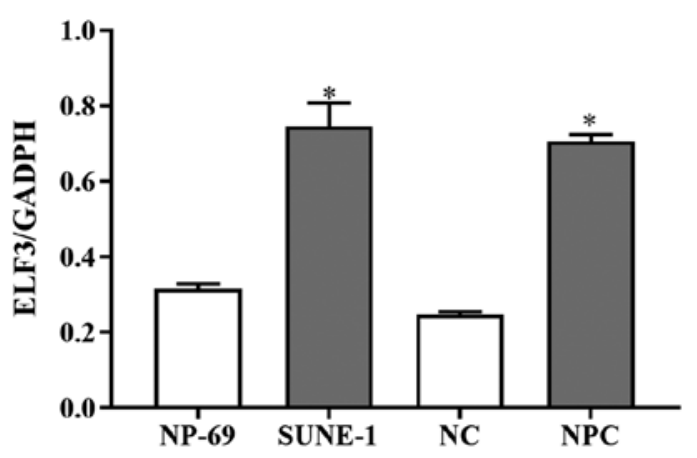

C

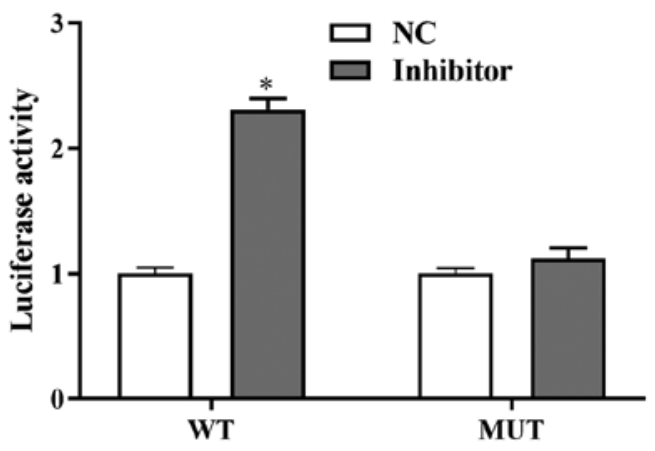

$\mathbf{E}$

ELF3

NP-69 SUNE-1 NC NPC

GAPDH
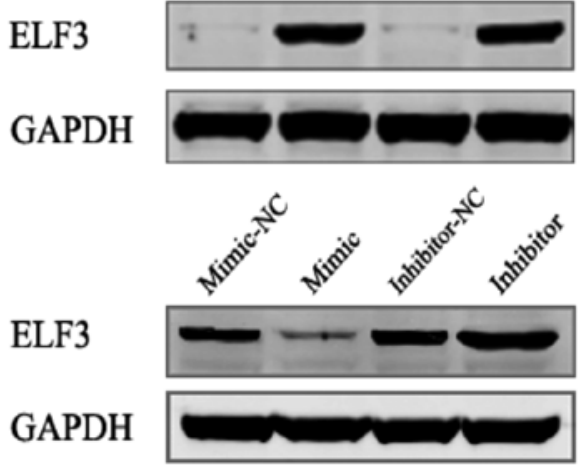

G

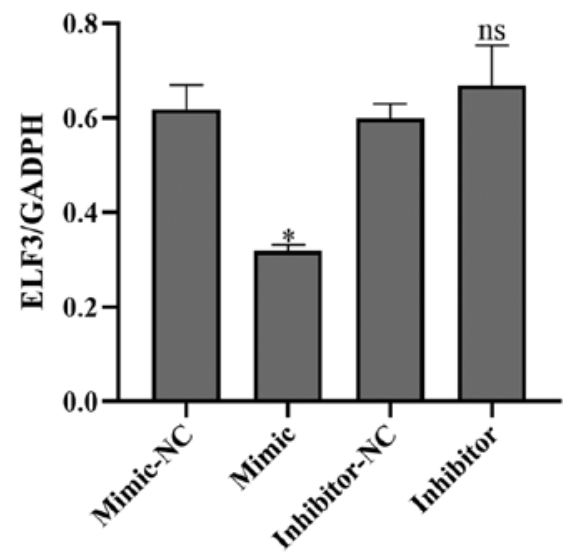

Figure 3. ELF3 is a direct target of miR-212. (A) Putative binding site of miR-212 at 333-340 bp of the 3'-UTR of ELF3. (B) Luciferase activity assay. miR-212 mimic significantly inhibited the luciferase activity of WT 3'-UTR of ELF3 but had no marked influence on MT 3'-UTR of ELF3. SUNE-1 cells were co-transfected with the WT or MT ELF3 3'-UTR construct, along with the miR-212 inhibitor or NC. At $48 \mathrm{~h}$ after transfection, the cells were lysed for the dual-luciferase reporter assay. (C) miR-212 inhibitor significantly increased the luciferase activity of WT 3'-UTR of ELF3 but had no marked influence on MT 3'-UTR of ELF3. (D) ELF3 expression fold change was detected by reverse transcription-quantitative PCR. (E) ELF3 protein expression levels detected by western blotting. (F) Western blot analysis using ImageJ v1.8.0 software for NPC tissues and SUNE-1 cells. (G) miR-212 mimic decreased ELF3 expression and miR-212 inhibitor increased ELF3 expression. " $\mathrm{P}<0.05,{ }^{* * *} \mathrm{P}<0.01$ vs. the respective control group. 3'-UTR, 3'-untranslated region; ELF3, E74-like factor 3; miR-212, microRNA-212; MT, mutant; NC, negative control; ns, not significant; WT, wild type. 
A

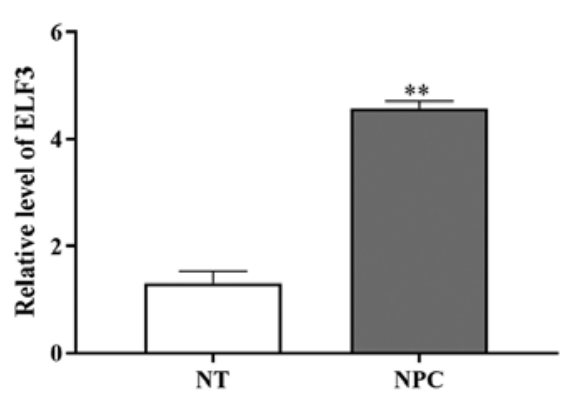

B

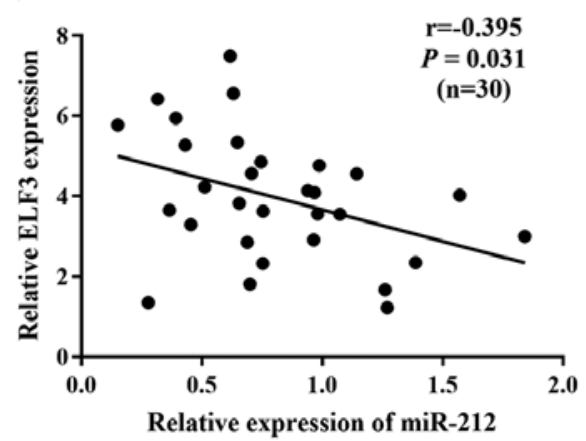

C

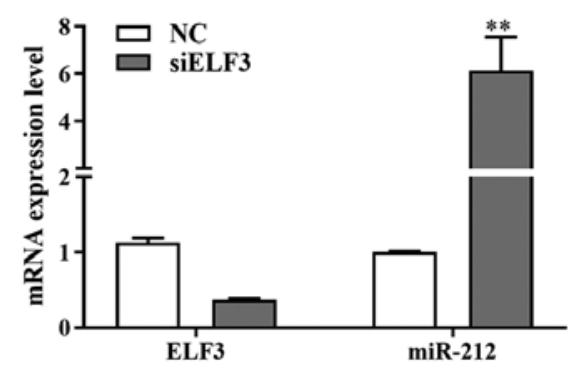

Figure 4. miR-212 expression is inversely correlated with the expression of ELF3. (A) ELF3 expression was significantly upregulated in NPC tissues compared with adjacent normal tissues (NT). (B) miR-212 expression was inversely correlated with the expression levels of ELF3. (C) miR-212 and ELF3 expression levels were detected by reverse transcription-quantitative PCR following transfection of SUNE-1 cells with ELF3 overexpression vectors, siRNA ELF3 and their respective negative controls. ${ }^{* *} \mathrm{P}<0.01$ vs. the respective control group. ELF3, E74-like factor 3; miR-212, microRNA-212; NC, negative control; si, small interfering RNA.

(Fig. 3C) and had no effect on the luciferase activity of the MT ELF3 3'-UTR.

Furthermore, the miR-212 mimic decreased the protein and mRNA expression levels of ELF3 and the miR-212 inhibitor increased the protein and mRNA expression levels of ELF3 compared with negative controls in SUNE-1 cells (Fig. 3D and E). Additionally, ImageJ software was used to assess ELF3 expression in NPC tissues and SUNE-1 cells (Fig. 3F and G). These results demonstrated that miR-212 could downregulate ELF3 by directly targeting its 3'-UTR.

miR-212 expression is inversely correlated with the expression level of ELF3. To investigate the expression levels of ELF3 in NPC, 30 freshly frozen NPC tissues and normal tissue specimens were analyzed. Using RT-qPCR, it was identified that the ELF3 expression was markedly increased in NPC tissues compared with normal adjacent tissues (Fig. 4A).

In addition, a negative correlation was revealed between the miR-212 and ELF3 expression levels in NPC tissues $(\mathrm{r}=-0.395 ; \mathrm{P}=0.031$; Fig. 4B). The downregulation of $\mathrm{miR}-212$ was found to inversely correlate with the clinical stage and local or distant metastasis, whereas the upregulation of ELF3 was directly associated with the clinical stage and local or distant metastasis in patients with HCC (Table I). miR-212 and ELF3 expression levels were detected by RT-qPCR following transfection of ELF3 overexpression vectors, ELF3 siRNA and their respective negative controls into SUNE-1 cells (Fig. 4C). Overexpression of ELF3 significantly reduced miR-212 mRNA expression, however transfection of siELF3 significantly increased miR-212 mRNA expression. These results indicated an inverse association between miR-212 and ELF3 expression in NPC.

\section{Discussion}

Dysregulation of mature miRNAs has been reported in numerous types of cancer. A number of studies have reported that miRNAs can serve roles as tumor suppressors and oncogenes. Aberrant expression levels of miRNAs and genes have been detected in NPC in a number of studies; several miRNAs are upregulated in NPC, including miR-27a-3p (21),
miR-93 (22) and miR-125b (23), and certain miRNAs are downregulated in NPC, including miR-135a (10), miR-203a-3p (12) and miR-185 (24). The present study demonstrated that the expression levels of miR-212 were significantly lower in NPC tissues and cell lines compared with normal controls. This indicated that miR-212 was downregulated in NPC, which is consistent with other studies regarding lung cancer (13), gastric cancer (14), hepatocellular carcinoma (25), prostate cancer (26) and NPC (15). The results of the present study suggested that miR-212 served as a tumor suppressor gene in NPC. In addition, aberrant expression of miR-212 has been demonstrated to regulate tumor migration and invasion (15). These results indicate that miR-212 may exert pivotal pathological and biological roles, which may contribute to the progression and growth of NPC.

miRNAs exert their roles by regulating downstream target genes via binding to the 3'-UTR. Several studies have revealed a number of target genes of miR-212, including SOX4 (13), paxillin (14), Kruppel like factor 4 (27) and SOX5 (28). The present study identified ELF3 as a direct target of miR-212 using a luciferase assay and bioinformatics analysis. miR-212 mimic markedly decreased the ELF3 expression level and miR-212 inhibitor increased the ELF3 expression level. In addition, transfection of SUNE-1 cells with siELF3 significantly reduced ELF3 mRNA expression, but increased miR-212 expression. Furthermore, it was demonstrated that the expression level of miR-212 was low in NPC cells and tissues, and miR-212 inhibited cell proliferation, possibly by targeting ELF3 in NPC. miR-212 expression was inversely correlated with ELF3 protein expression in NPC tissues. Additionally, the relative expression levels of ELF3 and miR-212 were associated with metastasis and TNM stage in NPC.

In conclusion, the present study provided an improved understanding regarding the pathogenesis of NPC. Increased miR-212 expression was identified to serve a positive role in NPC by inhibiting the proliferation of NPC cells via ELF3. This may provide a novel therapeutic target for NPC.

\section{Acknowledgements}

Not applicable. 


\section{Funding}

No funding was received.

\section{Availability of data and materials}

All datasets used and/or analyzed during the present study are available from the corresponding author on reasonable request.

\section{Authors' contributions}

YK performed the molecular biology experiments and drafted the manuscript. YC collected tissue samples and performed the statistical analysis. MT conceived the design of the study and drafted the manuscript. All authors read and approved the final manuscript.

\section{Ethics approval and consent to participate}

The human study was approved by the Ethics Committee of Rui Jin Hospital Affiliated to Shanghai Jiaotong University. (Shanghai, China). The patients provided written informed consent prior to the study.

\section{Patient consent for publication}

Not applicable.

\section{Competing interests}

The authors declare that they have no competing interests.

\section{References}

1. Han P, Wang X, Liang F, Liu Y, Qiu X, Xu Y, Chen R, Yu S and Huang X: Osteoradionecrosis of the skull base in nasopharyngeal carcinoma: Incidence and risk factors. Int J Radiat Oncol Biol Phys 102: 552-555, 2018

2. Lin K, Qiu F, Chen S, He X, Peng S and Chen H: Lack of association between the distribution of $\mathrm{ABO}$ blood groups and nasopharyngeal carcinoma in a population of Southern China. J Cancer Res Ther 14: 785-788, 2018.

3. Nakanishi Y, Wakisaka N, Kondo S, Endo K, Sugimoto H, Hatano M, Ueno T, Ishikawa K and Yoshizaki T: Progression of understanding for the role of Epstein-Barr virus and management of nasopharyngeal carcinoma. Cancer Metastasis Rev 36: 435-447, 2017.

4. Lin Z, Yang Z, He B, Wang D, Gao X, Tam SY and Wu VWC: Pattern of radiation-induced thyroid gland changes in nasopharyngeal carcinoma patients in 48 months after radiotherapy. PLoS One 13: e0200310, 2018.

5. Hezaveh K, Kloetgen A, Bernhart SH, Mahapatra KD, Lenze D, Richter J, Haake A, Bergmann AK, Brors B, Burkhardt B, et al: Alterations of microRNA and microRNA-regulated messenger RNA expression in germinal center B-cell lymphomas determined by integrative sequencing analysis. Haematologica 101: 1380-1389, 2016.

6. Dallaire A and Simard MJ: The implication of microRNAs and endo-siRNAs in animal germline and early development. Dev Biol 416: 18-25, 2016.

7. Chai C,Wu H,Wang B,EisenstatDD and Leng RP: MicroRNA-498 promotes proliferation and migration by targeting the tumor suppressor PTEN in breast cancer cells. Carcinogenesis 39: $1185-1196,2018$.

8. Gorbea C, Mosbruger T and Cazalla D: A viral Sm-class RNA base-pairs with mRNAs and recruits microRNAs to inhibit apoptosis. Nature 550: 275-279, 2017.
9. Chen D, Hu S, Wu Z, Liu J and Li S: The role of MiR-132 in regulating neural stem cell proliferation, differentiation and neuronal maturation. Cell Physiol Biochem 47: 2319-2330, 2018.

10. Wang LX, Kang ZP, Yang ZC, Ma RX, Tan Y, Peng XB, Dai RZ, Li J, Yu Y and Xu M: MicroRNA-135a inhibits nasopharyngeal carcinoma cell proliferation through targeting interleukin-17. Cell Physiol Biochem 46: 2232-2238, 2018.

11. Bao L, You B, Shi S, Shan Y, Zhang Q, Yue H, Zhang J, Zhang W, Shi Y, Liu Y, et al: Metastasis-associated miR-23a from nasopharyngeal carcinoma-derived exosomes mediates angiogenesis by repressing a novel target gene TSGA10. Oncogene 37: 2873-2889, 2018.

12. Jiang N, Jiang $X$, Chen Z, Song X, Wu L, Zong D, Song D, Yin L, Wang D, Chen C, et al: MiR-203a-3p suppresses cell proliferation and metastasis through inhibiting LASP1 in nasopharyngeal carcinoma. J Exp Clin Cancer Res 36: 138, 2017.

13. Tang T, Huan L, Zhang S, Zhou H, Gu L, Chen X and Zhang L: MicroRNA-212 functions as a tumor-suppressor in human non-small cell lung cancer by targeting SOX4. Oncol Rep 38: 2243-2250, 2017.

14. Li D, Li Z, Xiong J, Gong B, Zhang G, Cao C, Jie Z, Liu Y, Cao Y, Yan Y, et al: MicroRNA-212 functions as an epigenetic-silenced tumor suppressor involving in tumor metastasis and invasion of gastric cancer through down-regulating PXN expression. Am J Cancer Res 5: 2980-2997, 2015.

15. Jiang C, Wang H,Zhou L, Jiang T, Xu Y and Xia L: MicroRNA-212 inhibits the metastasis of nasopharyngeal carcinoma by targeting SOX4. Oncol Rep 38: 82-88, 2017.

16. Ma C, Nong K, Wu B, Dong B, Bai Y, Zhu H, Wang W, Huang X, Yuan Z and Ai K: miR-212 promotes pancreatic cancer cell growth and invasion by targeting the hedgehog signaling pathway receptor patched-1. J Exp Clin Cancer Res 33: 54, 2014.

17. Oliver JR, Kushwah R and Hu J: Multiple roles of the epithelium-specific ETS transcription factor, ESE-1, in development and disease. Lab Invest 92: 320-330, 2012.

18. Wang JL, Chen ZF, Chen HM, Wang MY, Kong X, Wang YC, Sun TT, Hong J, Zou W, Xu J and Fang JY: Elf3 drives $\beta$-catenin transactivation and associates with poor prognosis in colorectal cancer. Cell Death Dis 5: e1263, 2014.

19. Zheng L, Xu M, Xu J, Wu K, Fang Q, Liang Y, Zhou S, Cen D, Ji L, Han W and Cai X: ELF3 promotes epithelial-mesenchymal transition by protecting ZEB1 from miR-141-3p-mediated silencing in hepatocellular carcinoma. Cell Death Dis 9: 387, 2018.

20. Livak KJ and Schmittgen TD: Analysis of relative gene expression data using real-time quantitative PCR and the 2(-Delta Delta C(T)) method. Methods 25: 402-408, 2001.

21. Li L and Luo Z: Dysregulated miR-27a-3p promotes nasopharyngeal carcinoma cell proliferation and migration by targeting Mapk10. Oncol Rep 37: 2679-2687, 2017.

22. Zhang Y and Xu Z: miR-93 enhances cell proliferation by directly targeting CDKN1A in nasopharyngeal carcinoma. Oncol Lett 15: 1723-1727, 2018.

23. Li LN, Xiao T, Yi HM, Zheng Z, Qu JQ, Huang W, Ye X, Yi H, Lu SS, Li XH and Xiao ZQ: MiR-125b increases nasopharyngeal carcinoma radioresistance by targeting A20/NF- $\kappa$ B signaling pathway. Mol Cancer Ther 16: 2094-2106, 2017.

24. Cheng JZ, Chen JJ, Wang ZG and Yu D: MicroRNA-185 inhibits cell proliferation while promoting apoptosis and autophagy through negative regulation of TGF- $\beta 1 / \mathrm{mTOR}$ axis and HOXC6 in nasopharyngeal carcinoma. Cancer Biomark 23: 107-123, 2018.

25. Jia P, Wei G, Zhou C, Gao Q, Wu Y, Sun X and Li X: Upregulation of MiR-212 inhibits migration and tumorigenicity and inactivates Wnt/ $\beta$-catenin signaling in human hepatocellular carcinoma. Technol Cancer Res Treat 17: 1533034618765221, 2018.

26. Hu B, Jin X and Wang J: MicroRNA-212 targets mitogen-activated protein kinase 1 to inhibit proliferation and invasion of prostate cancer cells. Oncol Res 26: 1093-1102, 2018.

27. Song Y, Liu Y and Chen X: MiR-212 attenuates MPP+-induced neuronal damage by targeting KLF4 in SH-SY5Y cells. Yonsei Med J 59: 416-424, 2018.

28. Liu Y, Zhang XL, Li XF, Tang YC and Zhao X: miR-212-3p reduced proliferation, and promoted apoptosis of fibroblast-like synoviocytes via down-regulating SOX5 in rheumatoid arthritis. Eur Rev Med Pharmacol Sci 22: 461-471, 2018.

This work is licensed under a Creative Commons Attribution-NonCommercial-NoDerivatives 4.0 International (CC BY-NC-ND 4.0) License. 